(c) 2000 International Press

Adv. Theor. Math. Phys. 4 (2000) 1231-1257

\title{
Absence of Static, Spherically Symmetric Black Hole Solutions for Einstein-Dirac-Yang/Mills Equations with Complete Fermion Shells
}

\author{
Felix Finster \\ Max Planck Institute for Mathematics in the Sciences \\ Leipzig, Germany \\ Felix.Finster@mis.mpg.de \\ Joel Smoller \\ Mathematics Department \\ The University of Michigan, Ann Arbor, MI \\ smoller@umich.edu
}

Shing-Tung Yau

Mathematics Department

Harvard University, Cambridge, MA

yau@math.harvard.edu

\begin{abstract}
We study a static, spherically symmetric system of $(2 j+1)$ massive Dirac particles, each having angular momentum $j, j=1,2, \ldots$, in a classical gravitational and $S U(2)$ Yang-Mills field. We show that for any black hole solution of the associated Einstein-Dirac-Yang/Mills equations, the spinors must vanish identically outside of the event horizon.
\end{abstract}

e-print archive: http://xxx.lanl.gov/gr-qc/0005028 


\section{Introduction}

Recently the Einstein-Dirac-Yang/Mills (EDYM) equations were studied for a static, spherically symmetric system of a Dirac particle interacting with both a gravitational field and an $S U(2)$ Yang-Mills field $[1,2]$. In these papers, the Dirac particle had no angular momentum, and we could make a consistent ansatz for the Dirac wave function involving two real spinor functions. In the present paper, we allow the Dirac particles to have nonzero angular momentum $j, j=1,2, \ldots$ Similar to [3], we can build up a spherically symmetric system out of $(2 j+1)$ such Dirac particles. In this case however, a reduction to real 2-spinors is no longer possible, but we can obtain a consistent ansatz involving four real spinor functions.

We show that the only black hole solutions of our 4-spinor EDYM equations are those for which the spinors vanish identically outside the black hole; thus these EDYM equations admit only the Bartnik-McKinnon (BM) black hole solutions of the $S U(2)$ Einstein-Yang/Mills equations [4, 5]. This result extends our work in [2] to the case with angular momentum; it again means physically that the Dirac particles must either enter the black hole or escape to infinity. This generalization comes as a surprise because if one thinks of the classical limit, then classical point particles with angular momentum can "rotate around" the black hole on a stable orbit. Our result thus shows that the non-existence of black hole solutions is actually a quantum mechanical effect. A simple way of understanding the difference between classical and quantum mechanical particles is that for classical particles, the centrifugal barrier prevents the particles from falling into the black hole, whereas quantum mechanical particles can tunnel through this barrier. In our system, tunnelling alone does not explain the non-existence of black-hole solutions, because the Dirac particles are coupled to the classical fields; that is, they can influence the potential barrier. Our results are established by analyzing in detail the interaction between the matter fields and the gravitational field.

In Section 2, we derive the static, spherically symmetric $S U(2)$ EDYM equations with non-zero angular momentum of the Dirac particles. By assuming the BM ansatz for the YM potential (the vanishing of the electric component), the resulting system consists of 4 first-order equations for the spinors, two first-order Einstein equations, and a second-order equation for the YM potential. This EDYM system is much more complicated than the system considered in [2], and in order to make possible a rigorous mathematical analysis of the equations, we often assume (as in [3]) a power ansatz for the metric functions and the YM potential. Our analysis combines both geometrical and analytic techniques. 


\section{Derivation of the EDYM Equations}

We begin with the separation of variables for the Dirac equation in a static, spherically symmetric EYM background. As in [1], we choose the line element and the YM potential $\mathcal{A}$ in the form

$$
\begin{aligned}
d s^{2} & =\frac{1}{T(r)^{2}} d t^{2}-\frac{1}{A(r)} d r^{2}-r^{2} d \vartheta^{2}-r^{2} \sin ^{2} \vartheta d \varphi^{2} \\
\mathcal{A} & =w(r) \tau^{1} d \vartheta+\left(\cos \vartheta \tau^{3}+w(r) \sin \vartheta \tau^{2}\right) d \varphi
\end{aligned}
$$

with two metric functions $A, T$, and the YM potential $w$. The Dirac operator was computed in $[1$, Section 2] to be

$$
\begin{aligned}
G= & i T \gamma^{t} \partial_{t}+\gamma^{r}\left(i \sqrt{A} \partial_{r}+\frac{i}{r}(\sqrt{A}-1)-\frac{i}{2} \sqrt{A} \frac{T^{\prime}}{T}\right) \\
& +i \gamma^{\vartheta} \partial_{\vartheta}+i \gamma^{\varphi} \partial_{\varphi}+\frac{2 i}{r}(w-1)\left(\vec{\gamma} \vec{\tau}-\gamma^{r} \tau^{r}\right) \tau^{r} .
\end{aligned}
$$

This Dirac operator acts on 8-component wave functions, which as in [1] we denote by $\Psi=\left(\Psi^{\alpha u a}\right)_{\alpha, u, a=1,2}$, where $\alpha$ are the two spin orientations, $u$ corresponds to the upper and lower components of the Dirac spinor (usually called the "large" and "small" components, respectively), and $a$ is the YM index. The Dirac equation is

$$
(G-m) \Psi=0
$$

where $m$ is the rest mass of the Dirac particle, which we assume to be positive $(m>0)$.

As explained in [1], the Dirac operator (3) commutes with the "total angular momentum operators"

$$
\vec{J}=\vec{L}+\vec{S}+\vec{\tau}
$$

where $\vec{L}$ is angular momentum, $\vec{S}$ the spin operator, and $\vec{\tau}$ the standard basis of $\mathrm{su}(2)_{\mathrm{YM}}$. Thus the Dirac operator is invariant on the eigenspaces of total angular momentum, and we can separate out the angular dependence by restricting the Dirac operator to suitable eigenspaces of the operators $\vec{J}$. Since (5) can be regarded as the addition of angular momentum and two spins $\frac{1}{2}$, the eigenvalues of $\vec{J}$ are integers. In [1], the Dirac equation was considered on the kernel of the operator $J^{2}$; this leads to the twocomponent Dirac equation $[1,(2.23),(2.24)]$. Here we want to study the effect of angular momentum and shall thus concentrate on the eigenspaces of $J^{2}$ with eigenvalues $j(j+1), j=1,2, \ldots$ Since the eigenvalues of $J_{z}$ merely 
describe the orientation of the wave function in space, it is furthermore sufficient to restrict attention to the eigenspace of $J_{z}$ corresponding to the highest possible eigenvalue. Thus we shall consider the Dirac equation on the wave functions $\Psi$ with

$$
J^{2} \Psi=j(j+1) \Psi \quad \text { and } \quad J_{z} \Psi=j \Psi \quad(j=1,2, \ldots) .
$$

Since (6) involves only angular operators, it is convenient to analyze these equations on spinors $\Phi^{\alpha a}(\vartheta, \varphi)$ on $S^{2}$ (where $\alpha$ and $a$ are again the spin and YM indices, respectively). Let us first determine the dimension of the space spanned by the vectors satisfying (6). Using the well-known decomposition of two spins $\frac{1}{2}$ into a singlet and a triplet, we choose a spinor basis $\Phi_{s t}$ with $s=0,1$ and $-s \leq t \leq s$ satisfying

$$
(\vec{S}+\vec{\tau})^{2} \Phi_{s t}=s(s+1) \Phi_{s t}, \quad\left(S_{z}+\tau_{z}\right) \Phi_{s t}=t \Phi_{s t} .
$$

The spherical harmonics $\left(Y_{l k}\right)_{l \geq 0,-l \leq k \leq l}$, on the other hand, are a basis of $L^{2}\left(S^{2}\right)$. Using the rules for the addition of angular momentum[6], the wave functions satisfying (6) must be linear combinations of the following vectors,

$$
\begin{aligned}
& Y_{j j} \Phi_{00} \\
& Y_{j-1 j-1} \Phi_{11} \\
& Y_{j j-1} \Phi_{11} \quad, \quad Y_{j j} \Phi_{10} \\
& Y_{j+1 j-1} \Phi_{11}, \quad Y_{j+1 j} \Phi_{10}, \quad Y_{j+1 j+1} \Phi_{1-1} \text {. }
\end{aligned}
$$

These vectors all satisfy the second equation in (6), but they are not necessarily eigenfunctions of $J^{2}$. We now use the fact that a vector $\Psi \neq 0$ satisfying the equation $J_{z} \Psi=j \Psi$ is an eigenstate of $J^{2}$ with eigenvalue $j(j+1)$ if and only if it is in the kernel of the operator $J^{+}=J_{x}+i J_{y}$. Thus the dimension of the eigenspace (6) coincides with the dimension of the kernel of $J^{+}$, restricted to the space spanned by the vectors (7)-(10). A simple calculation shows that this dimension is four (for example, we have $J^{+}\left(Y_{j j-1} \Phi_{11}\right)=Y_{j j} \Phi_{11}=J^{+}\left(Y_{j j} \Phi_{10}\right)$, and thus $J^{+}$applied to the vectors (9) has a one-dimensional kernel).

We next construct a convenient basis for the angular functions satisfying (6). We denote the vector (7) by $\Phi_{0}$. It is uniquely characterized by the conditions

$$
\begin{aligned}
L^{2} \Phi_{0} & =j(j+1) \Phi_{0}, & L_{z} \Phi_{0} & =j \Phi_{0} \\
(\vec{S}+\vec{\tau}) \Phi_{0} & =0 & \left\|\Phi_{0}\right\|_{S^{2}} & =1 .
\end{aligned}
$$

We form the remaining three basis vectors by multiplying $\Phi_{0}$ with spherically symmetric combinations of the spin and angular momentum operators, 
namely

$$
\begin{aligned}
\Phi_{1} & =2 S^{r} \Phi_{0}=-2 \tau^{r} \Phi_{0} \\
\Phi_{2} & =\frac{2}{c}(\vec{S} \vec{L}) \Phi_{0}=-\frac{2}{c}(\vec{\tau} \vec{L}) \Phi_{0} \\
\Phi_{3} & =\frac{4}{c} S^{r}(\vec{S} \vec{L}) \Phi_{0}=-\frac{4}{c} \tau^{r}(\vec{\tau} \vec{L}) \Phi_{0}
\end{aligned}
$$

where

$$
c=\sqrt{j(j+1)} \neq 0 .
$$

Since the operators $S^{r}, \tau^{r}$, and $(\vec{S} \vec{L})$ commute with $\vec{J}$, it is clear that the vectors $\Phi_{1}, \ldots, \Phi_{3}$ satisfy (6). Furthermore, using the standard commutation relations between the operators $\vec{L}, \vec{x}$, and $\vec{S}$ [6], we obtain the relations

$$
\begin{aligned}
(\vec{S} \vec{L})^{2} & =\frac{1}{4} L^{2}+\frac{i}{2} \epsilon_{j k l} S_{l} L_{j} L_{k}=\frac{1}{4} L^{2}-\frac{1}{4} \epsilon_{j k l} S_{l} \epsilon_{j k m} L_{m} \\
& =\frac{1}{4} L^{2}-\frac{1}{2} \vec{S} \vec{L} \\
(\vec{S} \vec{\tau})^{2} & =\frac{1}{4} \tau^{2}-\frac{1}{2} \vec{S} \vec{\tau}=\frac{3}{16}-\frac{1}{2} \vec{S} \vec{\tau} \\
2 S^{r} \Phi_{0} & =-2 \tau^{r} \Phi_{0}=\Phi_{1} \\
2 S^{r} \Phi_{1} & =-2 \tau^{r} \Phi_{1}=\Phi_{0} \\
2 S^{r} \Phi_{2} & =2 \tau^{r} \Phi_{2}=\Phi_{3} \\
2 S^{r} \Phi_{3} & =2 \tau^{r} \Phi_{3}=\Phi_{2} \\
(\vec{S} \vec{\tau}) \Phi_{0} & =-S^{2} \Phi_{0}=-\frac{3}{4} \Phi_{0} \\
(\vec{S} \vec{\tau}) \Phi_{1} & =2 S_{k} \tau_{k} S^{r} \Phi_{0}=-2 S_{k}(\vec{x} \vec{S}) S_{k} \Phi_{0} \\
& =2 S^{2} S^{r} \Phi_{0}-S_{k} x_{k} \Phi_{0}=\frac{1}{2} S^{r} \Phi_{0}=\frac{1}{4} \Phi_{1} \\
(\vec{S} \vec{\tau}) \Phi_{2} & =\frac{2}{c}(\vec{S} \vec{\tau})(\vec{S} \vec{L}) \Phi_{0}=\frac{1}{c}(\vec{L} \vec{\tau}) \Phi_{0}-\frac{2}{c}(\vec{S} \vec{L})(\vec{S} \vec{\tau}) \Phi_{0} \\
& =-\frac{1}{c}(\vec{S} \vec{L}) \Phi_{0}+\frac{3}{2 c}(\vec{S} \vec{L}) \Phi_{0}=\frac{1}{4} \Phi_{2} \\
(\vec{S} \vec{\tau}) \Phi_{3} & =\frac{4}{c}(\vec{S} \vec{\tau}) S^{r}(\vec{S} \vec{\tau}) \Phi_{0}=\frac{2}{c} \tau^{r}(\vec{S} \vec{L}) \Phi_{0}-\frac{4}{c} S^{r}(\vec{S} \vec{\tau})^{2} \Phi_{0} \\
& =\frac{1}{2} \Phi_{3}-\frac{1}{2} S^{r} \Phi_{2}=\frac{1}{4} \Phi_{3} \\
(\vec{S} \vec{L}) \Phi_{0} & =\frac{c}{2} \Phi_{2} \\
(\vec{S} \vec{L}) \Phi_{1} & =2(\vec{S} \vec{L}) S^{r} \Phi_{0} \\
& =2 S_{j}\left[L_{j}, S^{r}\right] \Phi_{0}+2\left\{S_{j}, S^{r}\right\} L_{j} \Phi_{0}-2 S^{r}(\vec{S} \vec{L}) \Phi_{0} \\
& -2 i S_{j} \epsilon_{j k l} x_{k} S_{l} \Phi_{0}-\frac{c}{2} \Phi_{3} \\
& =1
\end{aligned}
$$




$$
\begin{aligned}
& =-\epsilon_{j k l} x_{j} \epsilon_{k l m} S_{m} \Phi_{0}-\frac{c}{2} \Phi_{3}=-\Phi_{1}-\frac{c}{2} \Phi_{3} \\
(\vec{S} \vec{L}) \Phi_{2} & =\frac{2}{c}(\vec{S} \vec{L})^{2} \Phi_{0}=\frac{2}{c}\left(\frac{1}{4} L^{2}-\frac{1}{2} \vec{S} \vec{L}\right) \Phi_{0}=\frac{c}{2} \Phi_{0}-\frac{1}{2} \Phi_{2} \\
(\vec{S} \vec{L}) \Phi_{3} & =\frac{4}{c}(\vec{S} \vec{L}) S^{r}(\vec{S} \vec{L}) \Phi_{0}=-\frac{2}{c} S^{r}(\vec{S} \vec{L}) \Phi_{0}-c S^{r} \Phi_{0} \\
& =-\frac{1}{2} \Phi_{3}-\frac{c}{2} \Phi_{1}
\end{aligned}
$$

and thus

$$
\begin{aligned}
2\left(\vec{S} \vec{\tau}-S^{r} \tau^{r}\right) \tau^{r} \Phi_{0} & =-\frac{1}{2} \Phi_{1} \\
2\left(\vec{S} \vec{\tau}-S^{r} \tau^{r}\right) \tau^{r} \Phi_{1} & =\frac{1}{2} \Phi_{0} \\
2\left(\vec{S} \vec{\tau}-S^{r} \tau^{r}\right) \tau^{r} \Phi_{2} & =0 \\
2\left(\vec{S} \vec{\tau}-S^{r} \tau^{r}\right) \tau^{r} \Phi_{3} & =0 .
\end{aligned}
$$

Using these relations, it is easy to verify that the vectors $\Phi_{0}, \ldots, \Phi_{3}$ are orthonormal on $L^{2}\left(S^{2}\right)$. We take for the wave function $\Psi$ the ansatz

$$
\begin{aligned}
& \Psi^{\alpha u a}(t, r, \vartheta, \varphi) \\
& =e^{-i \omega t} \frac{\sqrt{T(r)}}{r}\left(\alpha(r) \Phi_{0}^{\alpha a}(\vartheta, \varphi) \delta_{u, 1}+\gamma(r) \Phi_{2}^{\alpha a}(\vartheta, \varphi) \delta_{u, 1}\right. \\
& \left.\quad+i \beta(r) \Phi_{1}^{\alpha a}(\vartheta, \varphi) \delta_{u, 2}+i \delta(r) \Phi_{3}^{\alpha a}(\vartheta, \varphi) \delta_{u, 2}\right)
\end{aligned}
$$

with real functions $\alpha, \beta, \gamma$, and $\delta$, where $\omega>0$ is the energy of the Dirac particle. This is the simplest ansatz for which the Dirac equation (4) reduces to a consistent set of ODEs. Namely, we obtain the following system of ODEs for the four-component wave function $\Phi:=(\alpha, \beta, \gamma, \delta)$,

$$
\sqrt{A} \Phi^{\prime}=\left(\begin{array}{cccc}
\frac{w}{r} & -\omega T-m & \frac{c}{r} & 0 \\
\omega T-m & -\frac{w}{r} & 0 & -\frac{c}{r} \\
\frac{c}{r} & 0 & 0 & -\omega T-m \\
0 & -\frac{c}{r} & \omega T-m & 0
\end{array}\right) \Phi .
$$

Similar to [3], we consider the system of $(2 j+1)$ Dirac wave functions obtained from (11) by applying the ladder operators $J_{ \pm}$. Substituting the Einstein and YM equations [1] and using the ansatz (1) and (2), we get the 
following system of ODEs,

$$
\begin{aligned}
r A^{\prime}= & 1-A-\frac{1}{e^{2}} \frac{\left(1-w^{2}\right)^{2}}{r^{2}} \\
& -2 \omega T^{2}\left(\alpha^{2}+\beta^{2}+\gamma^{2}+\delta^{2}\right)-\frac{2 A w^{\prime 2}}{e^{2}} \\
\frac{2 r A}{T} T^{\prime}= & A-1+\frac{1}{e^{2}} \frac{\left(1-w^{2}\right)^{2}}{r^{2}} \\
& -2 \omega T^{2}\left(\alpha^{2}+\beta^{2}+\gamma^{2}+\delta^{2}\right)-\frac{2 A w^{\prime 2}}{e^{2}} \\
& +T\left[2 m\left(\alpha^{2}-\beta^{2}+\gamma^{2}-\delta^{2}\right)+\frac{4 c}{r}(\alpha \delta+\beta \gamma)+\frac{4 w}{r} \alpha \beta\right] \\
r^{2} A w^{\prime \prime}= & -w\left(1-w^{2}\right)+e^{2} r T \alpha \beta-\frac{1}{2} r^{2} A^{\prime} w^{\prime}+\frac{r^{2} A T^{\prime} w^{\prime}}{T} .
\end{aligned}
$$

Here (13) and (14) are the Einstein equations, and (15) is the YM equation. Notice that the YM equation does not depend on $\gamma$ and $\delta$; moreover the lower two rows in the Dirac equation (12) are independent of $w$. This means that the Dirac particles couple to the YM field only via the spinor functions $\alpha$ and $\beta$. Indeed, a main difficulty here as compared to the two-spinor problem [2] will be to control the behavior of $\gamma$ and $\delta$.

For later use, we also give the equations for the following composite functions,

$$
\begin{aligned}
r^{2}\left(A w^{\prime}\right)^{\prime}= & -w\left(1-w^{2}\right)+e^{2} r T \alpha \beta+\frac{1}{2} r^{2} \frac{\left(A T^{2}\right)^{\prime}}{T^{2}} w^{\prime} \\
r\left(A T^{2}\right)^{\prime}= & -4 \omega T^{4}\left(\alpha^{2}+\beta^{2}+\gamma^{2}+\delta^{2}\right)-\frac{4 A T^{2} w^{\prime 2}}{e^{2}} \\
& +T^{3}\left[2 m\left(\alpha^{2}-\beta^{2}+\gamma^{2}-\delta^{2}\right)+\frac{4 c}{r}(\alpha \delta+\beta \gamma)+\frac{4 w}{r} \alpha \beta\right]
\end{aligned}
$$

Also, it is quite remarkable and will be useful later that for $\omega=0$, the squared Dirac equation splits into separate equations for $(\alpha, \gamma)$ and $(\beta, \delta)$; namely from (12),

$$
\begin{aligned}
& \sqrt{A} \partial_{r}\left(\sqrt{A} \partial_{r} \Phi\right)=\left(m^{2}+\frac{c^{2}}{r^{2}}\right) \Phi \\
& +\left[\sqrt{A}\left(\begin{array}{cccc}
\left(\frac{w}{r}\right)^{\prime} & 0 & -\frac{c}{r^{2}} & 0 \\
0 & -\left(\frac{w}{r}\right)^{\prime} & 0 & \frac{c}{r^{2}} \\
-\frac{c}{r^{2}} & 0 & 0 & 0 \\
0 & \frac{c}{r^{2}} & 0 & 0
\end{array}\right)+\left(\begin{array}{cccc}
\frac{w^{2}}{r^{2}} & 0 & \frac{w c}{r^{2}} & 0 \\
0 & \frac{w^{2}}{r^{2}} & 0 & \frac{w c}{r^{2}} \\
\frac{w c}{r^{2}} & 0 & 0 & 0 \\
0 & \frac{w c}{r^{2}} & 0 & 0
\end{array}\right)\right] \Phi
\end{aligned}
$$




\section{Non-Existence Results}

As in [2], we consider the situation where $r=\rho>0$ is the event horizon of a black hole, i.e. $A(\rho)=0$, and $A(\rho)>0$ if $r>\rho$. We again make (cf. [1]) suitable assumptions on the regularity of the event horizon:

(A) The volume element $\sqrt{\mid \operatorname{det} g_{i j}}|=| \sin \vartheta \mid r^{2} A^{-1} T^{-2}$ is smooth and non-zero on the horizon; i.e.

$$
T^{-2} A^{-1}, T^{2} A \in C^{1}([\rho, \infty)) .
$$

(AII) The strength of the Yang-Mills field $F_{i j}$ is given by

$$
\operatorname{Tr}\left(F_{i j} F^{i j}\right)=\frac{2 A w^{\prime 2}}{r^{2}}+\frac{\left(1-w^{2}\right)^{2}}{r^{4}} .
$$

We assume that it is bounded near the horizon; i.e.

$$
w \text { and } A w^{\prime 2} \text { are bounded for } \rho<r<\rho+\varepsilon .
$$

Furthermore, the spinors should be normalizable outside and away from the event horizon, i.e.

$$
\int_{\rho+\varepsilon}^{\infty}|\Phi|^{2} \frac{T}{\sqrt{A}}<\infty \quad \text { for every } \varepsilon>0
$$

Finally, we assume that the metric functions and the YM potential satisfy a power ansatz near the event horizon. More precisely, setting

$$
u \equiv r-\rho,
$$

we assume the ansatz

$$
\begin{aligned}
A(r) & =A_{0} u^{s}+o\left(u^{s}\right) \\
w-w_{0} & =w_{1} u^{\kappa}+o\left(u^{\kappa}\right)
\end{aligned}
$$

with real coefficients $A_{0} \neq 0$ and $w_{1}$, powers $s, \kappa>0$ and $w_{0}=\lim _{r} \searrow \rho w(r)$. Here and in what follows,

$$
f(u)=o\left(u^{\nu}\right) \text { means that } \exists \delta>0 \text { with } \limsup _{r \searrow \rho}\left|u^{-\nu-\delta} f(u)\right|<\infty .
$$

Also, we shall always assume that the derivatives of a function in $o\left(u^{\nu}\right)$ have the natural decay properties; more precisely,

$$
f(u)=o\left(u^{\nu}\right) \quad \text { implies that } \quad f^{(n)}(u)=o\left(u^{\nu-n}\right) .
$$


According to $\mathbf{A}_{\mathbf{I}},(21)$ yields that $T$ also satisfies a power law, more precisely

$$
T(r) \sim u^{-\frac{s}{2}}+o\left(u^{-\frac{s}{2}}\right)
$$

Our main result is the following.

Theorem 3.1. Under the above assumptions, the only black hole solutions of the EDYM equations (12)-(15) are either the Bartnik-McKinnon black hole solutions of the EYM equations, or

$$
s=\frac{4}{3} \quad \text { and } \quad \kappa=\frac{1}{3} .
$$

In (24), the so-called exceptional case, the spinors behave near the horizon like

$$
(\alpha \beta)(r) \sim u^{\frac{1}{3}}+o\left(u^{\frac{1}{3}}\right), \quad 0<(\gamma \delta)(\rho)<\infty .
$$

Our method for the proof of this theorem is to assume a black hole solution with $\Phi \not \equiv 0$, and to show that this implies (24) and (25). The proof, which is split up into several parts, is given in Sections $4-7$.

In Section 8, we will analyze the exceptional case. It is shown numerically that the ansatz $(24),(25)$ does not yield global solutions of the EDYM equations. From this we conclude that for all black hole solutions of our EDYM system, the Dirac spinors must vanish identically outside of the event horizon.

\section{Proof that $\omega=0$}

Let us assume that there is a solution of the EDYM equations where the spinors are not identically zero, $\Phi \not \equiv 0$. In this section we will show that then $\omega$ must be zero. First we shall prove that the norm of the spinors $|\Phi|$ is bounded from above and below near the event horizon. We distinguish between the two cases where $A^{-\frac{1}{2}}$ is or is not integrable near the event horizon.

Lemma 4.1. If $A^{-\frac{1}{2}}$ is integrable near the event horizon $r=\rho$, then there are positive constant $k$ and $\varepsilon$ such that

$$
\frac{1}{k} \leq|\Phi(r)|^{2} \leq k, \quad \text { if } \rho<r<\rho+\varepsilon .
$$


Proof. Writing (12) as $\sqrt{A} \Phi^{\prime}=M \Phi$, we have

$$
\begin{aligned}
\frac{1}{2} & \sqrt{A} \frac{d}{d r}|\Phi|^{2}=\frac{1}{2} \Phi^{t}\left(M+M^{*}\right) \Phi \\
& =\frac{w}{r}\left(\alpha^{2}-\beta^{2}\right)-2 m(\alpha \beta+\gamma \delta)+\frac{2 c}{r}(\alpha \gamma-\beta \delta) \\
& \leq \frac{w}{r}\left(\alpha^{2}-\beta^{2}\right)+m\left(\alpha^{2}+\beta^{2}+\gamma^{2}+\delta^{2}\right)+\frac{2 c}{r}(\alpha \gamma-\beta \delta) \\
& \leq c_{1}|\Phi|^{2} .
\end{aligned}
$$

Here the constant $c_{1}$ is independent of $r \in(\rho, \rho+1]$, since $w$ is bounded near the horizon according to assumption $\mathbf{A}_{\mathbf{I I}}$. Since we are assuming that $\Phi \not \equiv 0$ in $r>\rho$, the uniqueness theorem for solutions of ODEs yields that $|\Phi|^{2}>0$ on $(\rho, \rho+1]$. Then dividing (27) by $\frac{1}{2} \sqrt{A}|\Phi|^{2}$ and integrating from $r_{1}$ to $r_{2}$, $\rho<r_{1}<r_{2}$, we get

$$
\left.|\log | \Phi\left(r_{2}\right)\right|^{2}-\log \left|\Phi\left(r_{1}\right)\right|^{2} \mid \leq 2 c_{1} \int_{r_{1}}^{r_{2}} A^{-\frac{1}{2}}(r) d r .
$$

Taking the limit $r_{1} \searrow \rho$ in this last inequality gives the desired result.

Lemma 4.2. If $A^{-\frac{1}{2}}$ is not integrable near the event horizon $r=\rho$ and $\omega \neq 0$, then there are positive constants $k$ and $\varepsilon$ such that

$$
\frac{1}{k} \leq|\Phi(r)|^{2} \leq k \quad \text { if } \rho<r<\rho+\varepsilon .
$$

Proof. Define the matrix $J$ by

$$
J=\left(\begin{array}{cccc}
1-\frac{m}{\omega T} & -\frac{w}{r \omega T} & 0 & -\frac{c}{r \omega T} \\
-\frac{w}{r \omega T} & 1+\frac{m}{\omega T} & -\frac{c}{r \omega T} & 0 \\
0 & -\frac{c}{r \omega T} & 1-\frac{m}{\omega T} & 0 \\
-\frac{c}{r \omega T} & 0 & 0 & 1+\frac{m}{\omega T}
\end{array}\right)
$$

and notice that, since $T(r) \rightarrow \infty$ as $r \searrow \rho, J$ is close to the identity matrix for $r$ near $\rho$. If we let

$$
F(r)=<\Phi(r), J(r) \Phi(r)>,
$$

then a straightforward calculation yields that

$$
F^{\prime}=<\Phi(r), J^{\prime}(r) \Phi(r)>.
$$


In a manner similar to that in [2], we can prove that $\left|J^{\prime}\right|$ is integrable near $r=\rho$, and as in [2], it follows that (28) holds.

Lemma 4.3. If $\Phi \not \equiv 0$ for $r>\rho$, then $\omega=0$.

Proof. Assume that $\omega \neq 0$. We write the $\left(A T^{2}\right)^{\prime}$ equation (17) as

$$
\begin{aligned}
& r\left(A T^{2}\right)^{\prime}=-4 \omega T^{4}|\Phi|^{2}-\frac{4 A w^{\prime 2}}{e^{2}} T^{2} \\
& +\left[2 m\left(\alpha^{2}-\beta^{2}+\gamma^{2}-\delta^{2}\right)+\frac{4 w}{r} \alpha \beta+\frac{4 c}{r}(\alpha \delta+\beta \gamma)\right] T^{3} .
\end{aligned}
$$

According to hypothesis $\mathbf{A}_{\mathbf{I I}}$, the left side of this equation is bounded near the event horizon. The Lemmas 4.1 and 4.2 together with $\mathbf{A}_{\mathbf{I I}}$ imply that the coefficients of $T^{4}, T^{3}$, and $T^{2}$ in this equation are all all bounded, and that the coefficient of $T^{4}$ is bounded away from zero near $r=\rho$. Assumption $\mathbf{A}_{\text {II }}$ implies that $T(r) \rightarrow \infty$ as $r \searrow \rho$. Hence the right side of (29) diverges as $r \searrow \rho$. This is a contradiction.

\section{$5 \quad$ Reduction to the Case $\alpha(\rho)=0, \beta(\rho) \neq 0$}

Since $\omega=0$, the Dirac equation (12) reduces to

$$
\sqrt{A} \Phi^{\prime}=\left(\begin{array}{cccc}
w / r & -m & c / r & 0 \\
-m & -w / r & 0 & -c / r \\
c / r & 0 & 0 & -m \\
0 & -c / r & -m & 0
\end{array}\right) \Phi \equiv M \Phi .
$$

The following Lemma gives some global information on the behavior of the solutions to (30).

Lemma 5.1. The function $(\alpha \beta+\gamma \delta)$ is strictly positive, decreasing, and tends to zero as $r \rightarrow \infty$.

Proof. A straightforward calculation gives

$$
\sqrt{A}(\alpha \beta+\gamma \delta)^{\prime}=-m|\Phi|^{2},
$$

so that $(\alpha \beta+\gamma \delta)(r)$ is a strictly decreasing function, and thus has a (possibly infinite) limit as $r \rightarrow \infty$. Since $|\Phi|^{2} \geq 2|\alpha \beta+\gamma \delta|$, we see that the 
normalization condition (20) holds only if this limit is zero. It follows that $(\alpha \beta+\gamma \delta)$ is strictly positive.

Next we want to show that the spinors have a (possibly infinite) limit as $r \searrow \rho$. When $A^{-\frac{1}{2}}$ is integrable near the event horizon, it is an immediate consequence of Lemma 4.1 that this limit exists and is even finite.

Corollary 5.2. If $A^{-\frac{1}{2}}$ is integrable near the horizon, then $\Phi$ has a finite limit for $r \searrow \rho$.

Proof. We can integrate (30) from $r_{1}$ to $r_{2}, \rho<r_{1}<r_{2}$,

$$
\Phi\left(r_{2}\right)-\Phi\left(r_{1}\right)=\int_{r_{1}}^{r_{2}} A^{-\frac{1}{2}}(r) M(r) \Phi(r) d r .
$$

Lemma 4.1 yields that the right side converges as $r_{1} \searrow \rho$, and hence $\Phi$ has a finite limit.

In the case when $A^{-\frac{1}{2}}$ is not integrable near the horizon, we argue as follows. According to the power ansatz (22), the matrix in (30) has a finite limit on the horizon. Exactly as shown in [7, Section 5] using the stable manifold theorem, there are fundamental solutions of the Dirac equation (that is, a basis of solutions of the ODE (30)) which behave near the event horizon exponentially like $\exp \left(\lambda_{j} \int A^{-\frac{1}{2}}\right)$, where $\lambda_{j} \in \mathbb{R}$ are the eigenvalues for $r \searrow \rho$ of the matrix in (30) (notice that the $\lambda_{j}$ are real since they are the eigenvalues of a symmetric matrix). Thus for any linear combination of these fundamental solutions, the spinor functions are monotone in a neighborhood of the event horizon, and hence as $r \searrow \rho, \Phi$ has a limit in $\mathbb{R} \cup\{ \pm \infty\}$. We set

$$
\Phi(\rho)=\lim _{r \searrow \rho} \Phi(r), \quad(\alpha \beta)(\rho)=\lim _{r \searrow \rho}(\alpha \beta)(r) .
$$

Proposition 5.3. $(\alpha \beta)(\rho)=0$.

Proof. We consider the $\left(A w^{\prime}\right)^{\prime}$ equation (16) with $\omega=0$,

$$
r^{2}\left(A w^{\prime}\right)^{\prime}=-w\left(1-w^{2}\right)+e^{2} r(\sqrt{A} T) \frac{\alpha \beta}{\sqrt{A}}+\frac{r^{2}\left(A T^{2}\right)^{\prime}}{2 A T^{2}} A w^{\prime} .
$$

Suppose that

$$
(\alpha \beta)(\rho)>0
$$


From hypotheses $\mathbf{A}_{\mathbf{I}}$ and and $\mathbf{A}_{\mathbf{I I}}$, we se that the coefficient of $\alpha \beta A^{-\frac{1}{2}}$ is positive near $r=\rho$, and the other terms on the right side of (31) are bounded. Thus we may write (31) in the form

$$
\left(A w^{\prime}\right)^{\prime}=\Phi(r)+\frac{\Psi((r)}{\sqrt{A(r)}},
$$

where $\Phi$ is bounded and $\Psi>0$ near $\rho$. Thus we can find constants $\Phi_{0}, \Psi_{0}$ satisfying

$$
\left(A w^{\prime}\right)^{\prime}>\Phi_{0}+\frac{\Psi_{0}}{\sqrt{A(r)}}, \quad \Psi_{0}>0
$$

for $r$ near $\rho$. Then exactly as in [2, Section 3], it follows that the spinors must vanish in $r>\rho$.

If on the other hand

$$
(\alpha \beta)(\rho)<0 .
$$

then (33) holds with $\Psi(r)<0$ near $\rho$. Thus

$$
-\left(A w^{\prime}\right)^{\prime}=-\Phi(r)-\frac{\Psi((r)}{\sqrt{A(r)}} .
$$

Setting $\tilde{w}=-w,(36)$ becomes

$$
\left(A \tilde{w}^{\prime}\right)^{\prime}=-\Phi(r)-\frac{\Psi((r)}{\sqrt{A(r)}},
$$

where $-\Psi(r)>0$ for $r$ near $\rho$. Thus we see that (33) holds for $w$ replaced by $\tilde{w}$. This again leads to a contradiction.

The next proposition rules out the case that both $\alpha$ and $\beta$ vanish on the event horizon.

Proposition 5.4. Either $\alpha(\rho)=0, \beta(\rho) \neq 0$ or $\alpha(\rho) \neq 0, \beta(\rho)=0$.

Proof. Suppose that

$$
\alpha(\rho)=0=\beta(\rho) .
$$

According to Lemma 5.1, $\gamma$ and $\delta$ cannot both vanish on the event horizon. Using (30), we have for $r$ near $\rho$,

$$
\begin{aligned}
\sqrt{A} \alpha^{\prime} & =\frac{c}{r} \gamma+o(1) \\
\sqrt{A} \beta^{\prime} & =-\frac{c}{r} \delta+o(1) .
\end{aligned}
$$


If $A^{-\frac{1}{2}}$ is not integrable near the event horizon, these equations show that $\gamma(\rho)$ and $\delta(\rho)$ are finite (otherwise multiplying (38) and (39) by $A^{-\frac{1}{2}}$ and integrating would contradict (37)); if $A^{-\frac{1}{2}}$ is integrable near $\rho$, Corollary 5.2 shows that $\gamma(\rho)$ and $\delta(\rho)$ are again finite.

From (17) with $\omega=0$ we have

$$
\begin{aligned}
r\left(A T^{2}\right)^{\prime}= & {\left[2 m\left(\alpha^{2}-\beta^{2}+\gamma^{2}-\delta^{2}\right)+\frac{4 w}{r} \alpha \beta+\frac{4 c}{r}(\alpha \delta+\beta \gamma)\right] T^{3} } \\
& -\frac{4}{e^{2}}\left(A w^{\prime 2}\right) T^{2}
\end{aligned}
$$

Since the coefficients of $T^{3}$ and $T^{2}$ are bounded, as is the left-hand side, we conclude that, since $T(r) \rightarrow \infty$ as $r \searrow \rho$, the coefficient of $T^{3}$ must vanish on the horizon,

$$
\left[2 m\left(\alpha^{2}-\beta^{2}+\gamma^{2}-\delta^{2}\right)+\frac{4 w}{r} \alpha \beta+\frac{4 c}{r}(\alpha \delta+\beta \gamma)\right]_{r=\rho}=0 .
$$

As a consequence, $\gamma(\rho)^{2}=\delta(\rho)^{2}$, and Lemma 5.1 yields that

$$
\gamma(\rho)=\delta(\rho) \neq 0
$$

Furthermore from (38) and (39), for $r$ near $\rho$,

$$
\operatorname{sgn} \alpha(r)=\operatorname{sgn} \gamma(r) \text { and } \operatorname{sgn} \beta(r)=-\operatorname{sgn} \delta(r) .
$$

From (42) and (43), we see that for $r$ near $\rho$, the spinors must lie in the shaded areas in one of the two configurations (I) or (II) in Figure 1. Now we claim that in either configuration (I) or (II), the shaded regions are invariant. For the proof, we consider the Dirac equation (30). One easily checks that the shaded regions in the $\alpha / \beta$-plots are invariant, provided that $\gamma$ and $\delta$ are as depicted in their shaded regions. Similarly, one verifies that the shaded regions in the $\gamma / \delta$-plots are invariant, provided that $\alpha$ and $\beta$ lie in the shaded regions. Moreover, Lemma 5.1 shows that the spinors cannot leave their regions simultaneously (i.e. for the same $r$ ). This proves the claim.

Next we consider the situation for large $r$. In the limit $r \rightarrow \infty$, the matrix $M$ in (30) goes over to the matrix $S$ given by

$$
S=\left(\begin{array}{cccc}
0 & -m & 0 & 0 \\
-m & 0 & 0 & 0 \\
0 & 0 & 0 & -m \\
0 & 0 & -m & 0
\end{array}\right)
$$


(I)
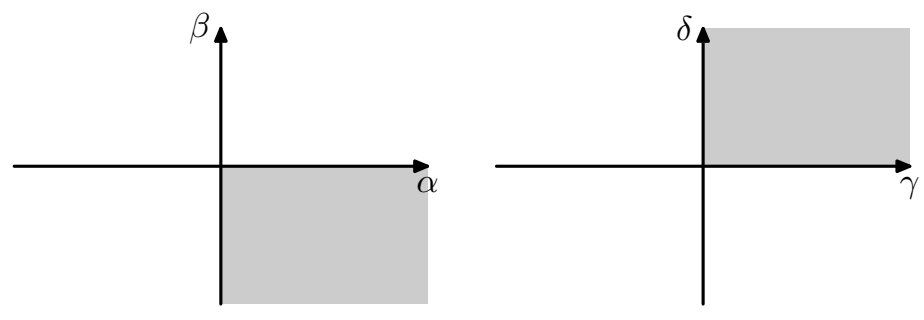

(II)
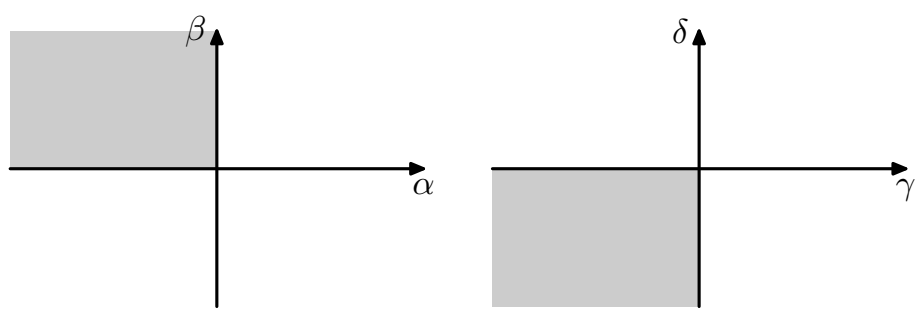

Figure 1: Invariant Regions for the Spinors

In $S$, the non-zero $2 \times 2$ upper and lower triangular blocks,

$$
\left(\begin{array}{cc}
0 & -m \\
-m & 0
\end{array}\right)
$$

have eigenvectors $(1,1)^{t}$ and $(1,-1)^{t}$ with corresponding eigenvalues $-m$ and $m$, respectively. Since the system of ODEs

$$
\sqrt{A} \Phi^{\prime}=S \Phi
$$

splits into separate equations for $(\alpha, \beta)$ and $(\gamma, \delta)$, we see that $(\alpha(r), \beta(r))$ must be a linear combination of $e^{-c(r) r}(1,1)^{t}$ and $e^{d(r) r}(1,-1)^{t}$, where the functions $c$ and $d$ are close to $m$. Since the spinors are assumed to be normalizable (i.e. (20) holds), and are non-zero for $r>\rho$, it follows that for large $r$, the spinors are close to a constant multiple of $e^{-c(r) r}(1,1)^{t}$, and thus for large $r, \operatorname{sgn} \alpha(r)=\operatorname{sgn} \beta(r)$. Similarly, for large $r, \operatorname{sgn} \gamma(r)=$ $\operatorname{sgn} \delta(r)$. This is a contradiction to the shaded invariant regions of Figure 1.

The two cases in Proposition 5.4 can be treated very similarly. Therefore we shall in what follows restrict attention to the first case. Furthermore, we know from Lemma 5.1 and Proposition 5.3 that $(\gamma \delta)(\rho)>0$. Using linearity of the Dirac equation, we can assume that both $\gamma(\rho)$ and $\delta(\rho)$ are positive. Hence the remaining problem is to consider the case where

$$
\alpha(\rho)=0, \quad \beta(\rho) \neq 0, \quad \gamma(\rho), \delta(\rho)>0 .
$$




\section{Proof that $A^{-\frac{1}{2}}$ is Integrable Near the Event Hori- zon}

In this section we shall assume that $A^{-\frac{1}{2}}$ is not integrable near the event horizon and deduce a contradiction. We work with the power ansatz (21),(22) and thus assume that $s \geq 2$.

We first consider the case $w_{0} \neq 0$. The first component of the squared Dirac equation (18) is

$$
\begin{aligned}
& \sqrt{A} \partial_{r}\left(\sqrt{A} \partial_{r} \alpha\right) \\
& =\left[m^{2}+\frac{c^{2}+w^{2}}{r^{2}}+\sqrt{A}\left(\frac{w}{r}\right)^{\prime}\right] \alpha+\frac{c(w-\sqrt{A})}{r^{2}} \gamma .
\end{aligned}
$$

The square bracket is bounded according to $\mathbf{A}_{\mathbf{I I}}$. Since $\alpha(\rho)=0$ and $\gamma(\rho)>$ 0 , our assumption $w_{0} \neq 0$ implies that the right side of $(45)$ is bounded away from zero near the event horizon, i.e. there are constants $\delta, \varepsilon$ with

$$
\pm \sqrt{A} \partial_{r}\left(\sqrt{A} \partial_{r} \alpha\right) \geq \delta \quad \text { for } \rho<r<\rho+\varepsilon
$$

where " \pm " corresponds to the two cases $w_{0}>1$ and $w_{0}<1$, respectively. We multiply this inequality by $A^{-\frac{1}{2}}$ and integrate from $r_{1}$ to $r_{2}, \rho<r_{1}<r_{2}$,

$$
\pm\left.\sqrt{A} \partial_{r} \alpha\right|_{r_{1}} ^{r_{2}} \geq \delta \int_{r_{1}}^{r_{2}} A^{-\frac{1}{2}}
$$

The right side diverges as $r_{1} \searrow \rho$, and thus $\lim _{r} \searrow \rho \sqrt{A} \partial_{r} \alpha=\mp \infty$. Hence near the event horizon, $\mp \partial_{r} \alpha \geq A^{-\frac{1}{2}}$, and integrating once again yields that $\lim _{r \searrow \rho} \alpha= \pm \infty$, in contradiction to $\alpha(\rho)=0$.

Suppose now that $w_{0}=0$. We first consider the $A$-equation (13), which since $\omega=0$ becomes

$$
r A^{\prime}=1-A-\frac{1}{e^{2}} \frac{\left(1-w^{2}\right)^{2}}{r^{2}}-\frac{2}{e^{2}} A w^{\prime 2} .
$$

Employing the power ansatz $(21),(22)$ gives

$$
O\left(u^{s-1}\right)=1+O\left(u^{s}\right)-\frac{1}{e^{2} r^{2}}+O\left(u^{2 \kappa}\right)+O\left(u^{s+2 \kappa-2}\right) .
$$

Here and in what follows,

$$
f(u)=O\left(u^{\nu}\right) \quad \text { means that } \quad \lim _{r \searrow \rho} u^{-\nu} f(u) \text { is finite and non-zero, }
$$


also we omit the expressions " $o\left(u^{\nu}\right)$." The constant term in (47) must vanish, and thus $e^{2} \rho^{2}=1$. Using also that $O\left(u^{s}\right)$ is of higher order, (47) reduces to

$$
O\left(u^{s-1}\right)=O(u)+O\left(u^{2 \kappa}\right)+O\left(u^{s+2 \kappa-2}\right) .
$$

Suppose first that $s>2$. Then (48) yields that $\kappa=\frac{1}{2}$. Substituting our power ansatz into the $A w^{\prime}$-equation (16) gives

$$
O\left(u^{s-\frac{3}{2}}\right)=O\left(u^{\frac{1}{2}}\right)+e^{2} r T \alpha \beta
$$

and thus $\alpha \beta=O\left(u^{\frac{1+s}{2}}\right)$. Since $\beta(\rho) \neq 0$, we conclude that there are constants $c_{1}, \delta>0$ with

$$
|\alpha| \leq c_{1} u^{\frac{1+s}{2}} \quad \text { for } \rho<r<\rho+\delta .
$$

From this one sees that the first summand on the right side of (45) is of higher order; more precisely,

$$
\sqrt{A} \partial_{r}\left(\sqrt{A} \partial_{r} \alpha\right)=O\left(u^{\frac{1}{2}}\right)
$$

Multiplying by $A^{-\frac{1}{2}}$ and integrating twice, we conclude that

$$
\alpha=O\left(u^{\frac{5}{2}-s}\right)
$$

and this contradicts (49).

The final case to consider is $w_{0}=0$ and $s=2$. Now the $A w^{\prime}$-equation (16) gives

$$
O\left(u^{\kappa}\right)=O\left(u^{\kappa}\right)+e^{2} T \alpha \beta
$$

and thus $\alpha=o(u)$. This gives a contradiction in (45) unless $w-\sqrt{A}=$ $o(u)$, and we conclude that $\kappa=1$. Now consider the Dirac equation (30). Since $w(\rho)=0$, the eigenvalues of the matrix in (30) on the horizon are $\lambda= \pm \sqrt{m^{2}+c^{2} / \rho^{2}}$. As a consequence, the fundamental solutions behave near the horizon $\sim u^{ \pm \sqrt{m^{2}+c^{2} / \rho^{2}}}$. The boundary conditions (44) imply that $\alpha \sim u^{+\sqrt{m^{2}+c^{2} / \rho^{2}}}$, whereas $\beta, \gamma, \delta \sim u^{-\sqrt{m^{2}+c^{2} / \rho^{2}}}$, and we conclude that

$$
(\alpha \delta+\beta \gamma)(\rho)>0
$$

Next we consider the $A T^{2}$-equation (17), which for $\omega=0$ takes the form (40). It is convenient to introduce for the square bracket the short notation

$$
[]=2 m\left(\alpha^{2}-\beta^{2}+\gamma^{2}-\delta^{2}\right)+\frac{4 w}{r} \alpha \beta+\frac{4 c}{r}(\alpha \delta+\beta \gamma) .
$$


We define the matrix $B$ by

$$
B=\left(\begin{array}{cccc}
m & w / r & 0 & c / r \\
w / r & -m & c / r & 0 \\
0 & c / r & m & 0 \\
c / r & 0 & 0 & -m
\end{array}\right)
$$

A short calculation shows that

$$
\text { [] }=2<\Psi, B \Psi>,
$$

and furthermore, using the Dirac equation (30),

$$
\begin{aligned}
{[]^{\prime} } & =2<\Psi, B^{\prime} \Psi>=4 \alpha \beta\left(\frac{w}{r}\right)^{\prime}-\frac{4 c}{r^{2}}(\alpha \delta+\beta \gamma) \\
& =\frac{4 w^{\prime}}{r} \alpha \beta-\frac{4 w}{r^{2}} \alpha \beta-\frac{4 c}{r^{2}}(\alpha \delta+\beta \gamma) .
\end{aligned}
$$

Since $(\alpha \beta)(\rho)=0$ and $(\alpha \delta+\beta \gamma)(\rho)>0$ according to $(50)$,

$$
-[]^{\prime} \geq c_{2} \quad \text { for } \rho<r<\rho+\delta
$$

and a constant $c_{2}>0$. Integrating on both sides shows that

$$
|[]| \geq c_{3} u \quad \text { for } \rho<r<\rho+\delta
$$

with $c_{3}>0$. As a consequence, the first summand in (40) diverges for $r \searrow \rho$, whereas the left side and the second summand on the right are bounded in this limit. This is a contradiction.

We conclude that $A^{-\frac{1}{2}}$ must be integrable near the event horizon, and so $s<2$.

\section{Proof of the Main Theorem}

In this section we shall analyze the EDYM equations with the power ansatz $(21),(22)$ near the event horizon. We will derive restrictions for the powers $s$ and $k$ until only the exceptional case (24) of Theorem 3.1 remains. So far, we know from Section 6 that $s<2$. A simple lower bound follows from the $A$-equation (13) which for $\omega=0$ simplifies to (46). Namely in view of hypothesis $\mathbf{A}_{\mathbf{I I}}$, the right-hand side of (46) is bounded, and thus $s \geq 1$. The case $s=1$ is excluded just as in [2] by matching the spinors across the horizon and applying a radial flux argument. Thus it remains only to consider $s$ in the range

$$
1<s<2 \text {. }
$$

We begin by deriving a power expansion for $\alpha$ near the event horizon. 
Lemma 7.1. Suppose that $w_{0} \neq 0$ or $\kappa \neq s / 2$. Then the function $\alpha$ behaves near the horizon as

$$
\alpha=\alpha_{0} u^{\sigma}+o\left(u^{\sigma}\right), \quad \alpha_{0} \neq 0
$$

where the power $\sigma$ is either

$$
\sigma=1-\frac{s}{2}
$$

or

$$
\sigma=\left\{\begin{array}{cc}
2-s & \text { if } w_{0} \neq 0 \\
2-s+\min (\kappa, s / 2) & \text { if } w_{0}=0
\end{array}\right.
$$

Proof. We set

$$
\sigma=\sup \left\{p: \limsup _{r \searrow \rho}\left|u^{-p} \alpha(r)\right|<\infty\right\} \leq \infty .
$$

Suppose first that $\sigma<\infty$. Then for every $\nu<\sigma$ there are constants $c>0$ and $\varepsilon>0$ with

$$
|\alpha(r)|<c u^{\nu} \quad \text { for } \rho<r<\rho+\varepsilon .
$$

We consider the first component of the squared Dirac equation (45) and write it in the form

$$
\sqrt{A} \partial_{r}\left(\sqrt{A} \partial_{r} \alpha\right)=f \alpha+g,
$$

where $f$ stands for the square bracket and $g$ for the last summand in (45), respectively. Multiplying by $A^{-\frac{1}{2}}$ and integrating gives

$$
\sqrt{A} \partial_{r} \alpha(r)=\int_{\rho}^{r} A^{-\frac{1}{2}}(f \alpha+g)+C
$$

with an integration constant $C$. We again multiply by $A^{-\frac{1}{2}}$ and integrate. Since $\alpha(\rho)=0$, we obtain

$$
\alpha(r)=\int_{\rho}^{r} A^{-\frac{1}{2}}(s) d s \int_{\rho}^{s} A^{-\frac{1}{2}}(f \alpha+g)+C \int_{\rho}^{r} A^{-\frac{1}{2}} .
$$

Note that the function $f$, introduced as an abbreviation for the square bracket in (45), is bounded near the horizon. Hence (58) yields a polynomial bound for $|f \alpha|$. Each multiplication with $A^{-\frac{1}{2}}$ and integration increases the power by $1-\frac{s}{2}$, and thus there is a constant $c_{1}$ with

$$
\int_{\rho}^{r} A^{-\frac{1}{2}}(s) d s \int_{\rho}^{s} A^{-\frac{1}{2}}|f \alpha| \leq c_{1} u^{2-s+\nu} \quad \text { for } \rho<r<\rho+\varepsilon .
$$


Since $2-s>0,(61)$ is of the order $o\left(u^{1-\frac{s}{2}+\nu}\right)$, and thus (60) can be written as

$$
\alpha(r)=\int_{\rho}^{r} A^{-\frac{1}{2}}(s) d s \int_{\rho}^{s} A^{-\frac{1}{2}} g+C \int_{\rho}^{r} A^{-\frac{1}{2}}+o\left(u^{1-\frac{s}{2}+\nu}\right) .
$$

Consider the behavior of the first two summands in (62). The function $g$ stands for the last summand in (45). If $w_{0} \neq 1$, it has a non-zero limit on the horizon. If on the other hand $w_{0}=1$, then $g \sim u^{\kappa}$. Substituting into (62) and integrating, one sees that the first summand in (62) is $\sim u^{\sigma}$ with $\sigma$ given by (56). The second summand in (62) vanishes if $C=0$, and is $\sim u^{\sigma}$ with $\sigma$ as in (55). According to (53), $1-\frac{s}{2}<2-s<2-s+\min (\kappa, s / 2)$. Thus the values of $\sigma$ in (55) and (56) are different, and so the first two summands in (62) cannot cancel each other. If we choose $\nu$ so large that $1-\frac{s}{2}+\nu \geq \sigma$, (62) yields the Lemma.

Suppose now that $\sigma$ given by (57) is infinite. Then choosing

$$
\nu=\max \left[1-\frac{s}{2}, 2-s+\min (\kappa, s / 2)\right]
$$

we see that the first two summands in (62) are of the order $O\left(u^{s}\right)$ with $s$ according to (55) and (56), respectively, and the last summand is of higher order. Thus (62) implies that $\sigma$ as defined by (57) is finite (namely, equal to the minimum of (55) and (56)), giving a contradiction. Thus $\sigma$ is indeed finite.

In the proof of Proposition 5.4, we already observed that the square bracket in the $A T^{2}$-equation (17) vanishes on the horizon (41). Let us now analyze this square bracket in more detail, where we use again the notation (51).

Proposition 7.2. $\kappa<1$ and

$$
[]=O\left(u^{\kappa+\sigma}\right)+O(u)
$$

with $\sigma$ as in Lemma 7.1.

Proof. The derivative of the square bracket is again given by (52). Now $\alpha_{0}=0, \beta_{0} \neq 0$ and from Lemma 5.1, $\alpha_{0} \delta_{0}+\beta_{0} \gamma_{0} \neq 0$; thus using (21), (22), and (54), we get, for $r$ near $\rho$,

$$
[]^{\prime}=O\left(u^{\kappa-1+\sigma}\right)+u^{\sigma+(\kappa)}+O(1),
$$


where we again omitted the expressions " $o(u)$ " and we use the notation

$$
(\kappa)=\left\{\begin{array}{ll}
\kappa & \text { if } w_{0}=0 \\
0 & \text { if } w_{0} \neq 0
\end{array} .\right.
$$

Integrating (64) and using that []$_{r=\rho}=0$ according to (41), we obtain that

$$
[]=O\left(u^{\kappa+\sigma}\right)+u^{\sigma+(\kappa)+1}+O(u) \text {. }
$$

Suppose $\kappa \geq 1$. Then $\kappa+\sigma>1$ and $\sigma+(\kappa)+1>1$, and (65) becomes

$$
\text { [] }=O(u) \text {. }
$$

We write the $A T^{2}$-equation (40) as

$$
r\left(A T^{2}\right)^{\prime}=T^{3}[]-\frac{4 A T^{2} w^{\prime 2}}{e^{2}} .
$$

Since $\left(A T^{2}\right)^{\prime}$ is bounded and $T^{3}=O\left(u^{-\frac{3 s}{2}}\right)$ (by virtue of hypothesis $\mathbf{A}_{\mathbf{I}}$ ), (66) behaves near the event horizon like

$$
u^{0}=O\left(u^{1-\frac{3 s}{2}}\right)+O\left(u^{2 \kappa-2}\right) .
$$

Since $2 \kappa-2 \geq 0$ and $1-\frac{3 s}{2}<0$, the right side of (67) is unbounded as $r \searrow \rho$, giving a contradiction. We conclude that $\kappa<1$.

For $\kappa<1$, the second summand in (65) is of higher order than the first, and we get (63).

In the remainder of this section, we shall substitute the power expansions (21)-(23) and (54) into the EDYM equations and evaluate the leading terms (i.e. the lowest powers in $u$ ). This will amount to a rather lengthy consideration of several cases, each of which has several subcases. We begin with the case $w_{0} \neq 0, \pm 1$. The $A$-equation (13) simplifies to (46). The $A T^{2}$-equation (17) for $\omega=0$ takes the form (40), and we can for the square bracket use the expansion of Proposition 7.2. Finally, we also consider the $A w^{\prime}$-equation (16). Using the regularity assumption $\mathbf{A}_{\mathbf{I}}$, we obtain

$$
\begin{array}{cl}
A \text {-eqn: } & O\left(u^{s-1}\right)=1-\frac{\left(1-w_{0}^{2}\right)^{2}}{e^{2} \rho^{2}}+O\left(u^{\kappa}\right)+O\left(u^{s+2 \kappa-2}\right) \\
A T^{2} \text {-eqn: } & u^{0}=O\left(u^{\kappa+\sigma-\frac{3 s}{2}}\right)+O\left(u^{1-\frac{3 s}{2}}\right)+O\left(u^{2 \kappa-2}\right) \\
A w^{\prime} \text {-eqn: } & O\left(u^{s+\kappa-2}\right)=w_{0}\left(1-w_{0}^{2}\right)+O\left(u^{\kappa}\right)+O\left(u^{\sigma-\frac{s}{2}}\right) .
\end{array}
$$


First consider (68). According to $\mathbf{A}_{\mathbf{I I}}, s+2 \kappa-2 \geq 0$, and so all powers in (68) are positive. We distinguish between the cases where the power $s+2 \kappa-2$ is larger, smaller, or equal to the other powers on the right of (68). Making sure in each case that the terms of leading powers may cancel each other, we obtain the cases and conditions

$$
\begin{aligned}
& \text { (a) } \kappa<s+2 \kappa-2 \Longrightarrow w_{0}^{2}=1 \pm e \rho, \kappa=s-1, s \geq \frac{3}{2} \\
& \text { (b) } \kappa=s+2 \kappa-2 \Longrightarrow w_{0}^{2}=1 \pm e \rho, \kappa=2-s, s \geq \frac{3}{2} \\
& \text { (c) } \kappa>s+2 \kappa-2>0 \Longrightarrow w_{0}^{2}=1 \pm e \rho, \kappa=\frac{1}{2}, s<\frac{3}{2} \\
& \text { (d) } \quad s+2 \kappa-2=0 \Longrightarrow \kappa=1-\frac{s}{2} .
\end{aligned}
$$

In Case (a), the relations in (71) imply that

$$
1-\frac{3 s}{2}<2 s-4=2 \kappa-2 .
$$

Hence (69) yields $1-3 s / 2=\kappa+\sigma-3 s / 2$, so

$$
\sigma=1-\kappa=2-s .
$$

This is consistent with Lemma 7.1. But we get a contradiction in (70) as follows. Since $\kappa=s-1$, we have $s+\kappa-2=2 s-3>0$; on the other hand,

$$
\sigma-\frac{s}{2}=2-s-\frac{s}{2}=2-\frac{3 s}{2}<0 \text {. }
$$

Thus the left-hand side of (70) is bounded, but the right-hand side is unbounded as $r \searrow \rho$. This completes the proof in Case (a).

In Case (b), (70) yields that

$$
\sigma \geq \frac{s}{2}
$$

We consider the two cases (55) and (56) in Lemma 7.1. In the first case, (76) yields that $s \leq 1$, contradicting (53). In the second case, (76) implies that $s \leq \frac{4}{3}$. This contradicts the inequality in (72), and thus completes the proof in Case (b).

In Case (c), the relations in (73) give $s+\kappa-2=s-\frac{3}{2}<0$, and thus (70) implies that $s+\kappa-2=\sigma-\frac{s}{2}$, so $\sigma=\frac{3}{2}(s-1)$. According to Lemma 7.1, $\sigma=2-s$ or $\sigma=1-\frac{s}{2}$. In the first of these cases, we conclude that $s=\frac{7}{5}$ and $\sigma=\frac{3}{5}$. Substituting these powers into (69), we get

$$
u^{0}=O\left(u^{-1}\right)+O\left(u^{-\frac{11}{10}}\right)+O\left(u^{-1}\right),
$$


which clearly yields a contradiction. Thus $\sigma=1-\frac{s}{2}$, giving

$$
s=\frac{5}{4}, \quad \kappa=\frac{1}{2}, \quad \sigma=\frac{3}{8} .
$$

This case is ruled out in Lemma 7.3 below.

In Case (d), we consider (70). Since $s+\kappa-2=-\kappa<0$, we obtain that $s+\kappa-2=\sigma-\frac{\sigma}{2}$ and thus $\sigma=s-1$. Lemma 7.1 yields the two cases

$$
\begin{aligned}
s & =\frac{4}{3}, & \kappa & =\frac{1}{3}, & \sigma & =\frac{1}{3} \quad \text { and } \\
s & =\frac{3}{2}, & \kappa & =\frac{1}{4}, & \sigma & =\frac{1}{2} .
\end{aligned}
$$

The first of these cases is the exceptional case of Theorem 3.1, and the second case is excluded in Lemma 7.4 below. This concludes the proof of Theorem 3.1 in the case $w_{0} \neq 0, \pm 1$.

We next consider the case $\underline{w_{0}= \pm 1}$. Then the expansions (68)-(70) must be modified to

$$
\begin{aligned}
A \text {-eqn: } & O\left(u^{s-1}\right)=1+O\left(u^{2 \kappa}\right)+O\left(u^{s+2 \kappa-2}\right) \\
A T^{2} \text {-eqn: } & u^{0}=O\left(u^{\kappa+\sigma-\frac{3 s}{2}}\right)+O\left(u^{1-\frac{3 s}{2}}\right)+O\left(u^{2 \kappa-2}\right) \\
A w^{\prime} \text {-eqn: } & O\left(u^{s+\kappa-2}\right)=O\left(u^{\kappa}\right)+O\left(u^{\sigma-\frac{s}{2}}\right) .
\end{aligned}
$$

One sees immediately that, in order to compensate the constant term in (80), $s+2 \kappa-2$ must be zero. Hence $s+\kappa-2=-\kappa<0$, and (82) yields that $s+\kappa-2=\sigma-\frac{s}{2}$ and thus $\sigma=s-1$. Now consider Lemma 7.1. In case (55), we get the exceptional case of Theorem 3.1, whereas case (56) yields that

$$
s=\frac{3}{2}, \quad \kappa=\frac{1}{4}, \quad \sigma=\frac{1}{2} .
$$

This case is ruled out in Lemma 7.4 below, concluding the proof of Theorem 3.1 in the case $w_{0}= \pm 1$.

The final case to consider is $\underline{w_{0}=0}$. In this case, the expansions corresponding to (68)-(70) are

$$
\begin{array}{cl}
A \text {-eqn: } & O\left(u^{s-1}\right)=1-\frac{1}{e^{2} \rho^{2}}+O\left(u^{2 \kappa}\right)+O\left(u^{s+2 \kappa-2}\right) \\
A T^{2} \text {-eqn: } & u^{0}=O\left(u^{\kappa+\sigma-\frac{3 s}{2}}\right)+O\left(u^{1-\frac{3 s}{2}}\right)+O\left(u^{2 \kappa-2}\right) \\
A w^{\prime} \text {-eqn: } & O\left(u^{s+\kappa-2}\right)=O\left(u^{\kappa}\right)+O\left(u^{\sigma-\frac{s}{2}}\right) .
\end{array}
$$

If $s+2 \kappa-2=0$, we obtain exactly as in the case $w_{0} \neq 0, \pm 1$ above that $\sigma=s-1$. It follows that $\kappa>\frac{s}{2}$, and Lemma 7.1 yields either the exceptional 
case of Theorem 3.1, or $s=2$, contradicting (53). If on the other hand $s+2 \kappa-2>0$, we can in (83) use the inequality $s+2 \kappa-2<2 \kappa$ to conclude that $s-1=s+2 \kappa-2$ and thus $\kappa=\frac{1}{2}$. Now $\kappa<\frac{s}{2}$, and Lemma 7.1 together with (85) yields the two cases

$$
\begin{aligned}
s & =\frac{5}{4}, & \kappa & =\frac{1}{2}, & \sigma & =\frac{3}{8} \\
s & =\frac{8}{5}, & \kappa & =\frac{1}{2}, & \sigma & =\frac{9}{10} .
\end{aligned}
$$

The first case is ruled out in Lemma 7.3 below, whereas the second case leads to a contradiction in (84). This concludes the proof of Theorem 3.1, except for the special cases treated in the following two lemmas.

Lemma 7.3. There is no solution of the EDYM equations satisfying the power ansatz (21), (22), and (54) with

$$
s=\frac{5}{4}, \quad \kappa=\frac{1}{2}, \quad \sigma=\frac{3}{8} .
$$

Proof. Suppose that there is a solution of the EDYM equations with

$$
\begin{aligned}
& A(r)=A_{0} u^{\frac{5}{4}}+o\left(u^{\frac{5}{4}}\right) \\
& w(r)=w_{1} u^{\frac{1}{2}}+o\left(u^{\frac{1}{2}}\right)
\end{aligned}
$$

with parameters $A_{0}, w_{1} \neq 0$. Consider the $A$-equation (46). The left side is of the order $(r-\rho)^{\frac{1}{4}}$. Thus the constant terms on the right side must cancel each other. Then the right side is also of the order $u^{\frac{1}{4}}$. Comparing the coefficients gives

$$
\frac{5}{4} \rho A_{0}=-\frac{1}{2 e^{2}} A_{0} w_{1}^{2} .
$$

This equation yields a contradiction because both sides have opposite sign.

Lemma 7.4. There is no solution of the EDYM equations satisfying the power ansatz (21), (22), and (54) with

$$
s=\frac{3}{2}, \quad \kappa=\frac{1}{4}, \quad \sigma=\frac{1}{2} .
$$

Proof. According to (21), we can write the function $\sqrt{A}$ as

$$
\sqrt{A}=u^{\frac{3}{4}}+f(u) \quad \text { with } \quad f=o\left(u^{\frac{3}{4}}\right) .
$$


Employing the ansatz (21),(22) into the A-equation (46), one sees that

$$
A w^{\prime 2}=u^{0}+u^{\frac{1}{4}}+o\left(u^{\frac{1}{4}}\right) .
$$

We solve for $\left(w^{\prime}\right)^{-1}$ and substitute (87) to obtain

$$
\frac{1}{w^{\prime}}=u^{\frac{3}{4}}+u+c_{1} f+o(u)
$$

with a real constant $c_{1}$. Now consider the $A T^{2}$-equation (40), which we write again in the form (66) and multiply by $A$,

$$
r A\left(A T^{2}\right)^{\prime}=\left(A T^{2}\right)^{\frac{3}{2}} A^{-\frac{1}{2}}[]-\frac{4}{e^{2}}\left(A T^{2}\right)\left(A w^{\prime 2}\right) .
$$

As in the proof of Proposition 7.2, a good expansion for the square bracket is obtained by integrating its derivative. Namely, according to (52),

$$
[]^{\prime}=\frac{4}{r} w^{\prime} \alpha \beta+u^{0}+o\left(u^{0}\right)
$$

and thus

$$
A^{-\frac{1}{2}}(r)[]=\frac{4}{r} A^{-\frac{1}{2}} \int_{\rho}^{r}\left(w^{\prime} \alpha \beta\right)(s) d s+u^{\frac{1}{4}}+o\left(u^{\frac{1}{4}}\right) .
$$

Substituting into (90) and using $\mathbf{A}_{\mathbf{I}}$ and (88), we obtain

$$
A^{-\frac{1}{2}}(r) \int_{\rho}^{r} w^{\prime} \alpha \beta=u^{0}+u^{\frac{1}{4}}+o\left(u^{\frac{1}{4}}\right) .
$$

We multiply by $\sqrt{A}$, substitute $(87)$ and differentiate,

$$
w^{\prime} \alpha \beta=u^{-\frac{1}{4}}+u^{0}+c_{2} f^{\prime}+o\left(u^{0}\right) .
$$

Multiplying by $1 / w^{\prime}$ and using (89) gives the following expansion for $\alpha \beta$,

$$
\alpha \beta=u^{\frac{1}{2}}+u^{\frac{3}{4}}+c_{3} u^{\frac{3}{4}} f^{\prime}+c_{4} u^{-\frac{1}{4}} f+o\left(u^{\frac{3}{4}}\right) .
$$

Next we multiply the $A w^{\prime}$-equation (16) by $\sqrt{A}$ and write it as

$$
r^{2} \sqrt{A}\left(\sqrt{A}\left(\sqrt{A} w^{\prime}\right)\right)^{\prime}=e^{2} r(\sqrt{A} T)(\alpha \beta)+u^{\frac{3}{4}}+o\left(u^{\frac{3}{4}}\right) .
$$

We apply $\mathbf{A}_{\mathbf{I}}$ and substitute (87), (88), and (91). This gives an equation of the form (modulo higher order terms),

$$
u^{\frac{1}{2}}+u^{\frac{3}{4}}+u^{\frac{3}{4}} f^{\prime}+u f^{\prime}+u^{-\frac{1}{4}} f+u^{0}=u^{\frac{1}{2}}+u^{\frac{3}{4}}+u^{\frac{3}{4}} f^{\prime}+u^{-\frac{1}{4}} f .
$$


The constant term $\sim u^{0}$ must vanish since all the other terms tend to zero as $u \rightarrow 0$. Furthermore, the $u^{\frac{1}{2}}$ terms must cancel because all the other terms are $o\left(u^{\frac{1}{2}}\right)$. We thus obtain

$$
u^{\frac{3}{4}} f^{\prime}+u^{-\frac{1}{4}} f=u^{\frac{3}{4}}+u f^{\prime}+f,
$$

so that

$$
u f^{\prime}+f=u+u^{\frac{5}{4}} f^{\prime}+u^{\frac{1}{4}} f=u+o(u),
$$

and we find that $f$ satisfies an equation of the form

$$
d_{1} u f^{\prime}+d_{2} f=d_{3} u+o(u) .
$$

A straightforward but tedious calculation yields that the coefficients $d_{1}$ and $d_{2}$ both vanish, and that $d_{3}$ is non-zero. This is a contradiction.

\section{The Exceptional Case}

In this section, we consider the exceptional case

$$
s=\frac{4}{3}, \quad \kappa=\frac{1}{3}, \quad \sigma=\frac{1}{3} .
$$

By employing the power ansatz (21), (22), and (54) into the EDYM equations and comparing coefficients (using Mathematica), we find that the solution near the event horizon is determined by the five free parameters $\left(\beta_{0}, \gamma_{0}, m, c, \rho\right)$. The remaining parameters are given by

$$
\begin{aligned}
\delta_{0} & =\sqrt{\gamma_{0}^{2}-\beta_{0}^{2}+\frac{2 c}{r m} \beta_{0} \gamma_{0}} \\
w_{0} & =-\frac{c \delta_{0}}{\beta_{0}} \\
A_{0} & =\frac{9 \beta_{0}}{r} \sqrt{\frac{m^{2} r^{2} \beta_{0}^{2}-2 c m r \beta_{0} \gamma_{0}+c^{2} \gamma_{0}^{2}}{r^{2}-\left(1-w_{0}^{2}\right)^{2}}} \\
\alpha_{0} & =-3 \frac{m r \beta_{0}-c \gamma_{0}}{r \sqrt{A_{0}}} \\
w_{1} & =\frac{9 \alpha_{0} \beta_{0}}{2 r A_{0}}
\end{aligned}
$$

Expanding to higher order, we obtain after an arduous calculation two further constraints on the free parameters, thus reducing the problem to one 
involving only three parameters. We investigated this three-parameter space numerically starting at $r_{0}=\rho+\varepsilon$ and found strong evidence that no global black hole solutions exist. Indeed, either the power ansatz was inconsistent near the event horizon (that is, for $r$ close to $r_{0}+\varepsilon$ the numerical solution deviated from the power ansatz, and became singular as $\varepsilon \searrow 0$ ), or else the solutions developed a singularity for finite $r$.

Acknowledgments: JS would like to thank the Max Planck Institute for Mathematics in the Sciences, Leipzig, and in particular Professor E. Zeidler, for their hospitality and generous support. FF and JS are grateful to the Harvard University Mathematics Department for support.

The research of JS and STY was supported in part by the NSF, Grant No. DMS-G-9802370 and 33-585-7510-2-30.

\section{References}

[1] F. Finster, J. Smoller, and S.-T. Yau, "The interaction of Dirac particles with non-abelian gauge fields and gravity - bound states," grqc/0001067, Nucl. Phys. B 584 (2000) 387-414

[2] F. Finster, J. Smoller, and S.-T. Yau, "The interaction of Dirac particles with non-abelian gauge fields and gravity - black holes," gr-qc/9910047, Mich. Math. J. 47 (2000) 199-208

[3] Finster, F., Smoller, J., and Yau, S.-T., "Non-existence of black hole solutions for a spherically symmetric, static Einstein-Dirac-Maxwell system," gr-qc/9810048, Commun. Math. Phys. 205 (1999) 249-262

[4] Kunzle, H.P. and Masood-ul-Alam, A.K.M., "Spherically symmetric static SU(2) Einstein-Yang-Mills fields," J. Math. Phys. 31 (1990) 928935

[5] Smoller, J., Wasserman, A. and Yau, S.-T., "Existence of black hole solutions for the Einstein-Yang/Mills equations," Commun. Math. Phys. 154 (1993) 377-401

[6] Landau, L.D., Lifshitz, E.M., "Quantum Mechanics," Pergamon Press (1977)

[7] Finster, F., Smoller, J., and Yau, S.-T., "Non-existence of time-periodic solutions of the Dirac equation in a Reissner-Nordström black hole background," gr-qc/9805050, J. Math. Phys. 41 (2000) 2173-2194 